\title{
Massive left upper abdominal cyst: how to diagnose and what to do
}

\author{
J Jacob, ${ }^{1}$ (D) N Patel ${ }^{2}$ iD \\ ${ }^{1}$ Department of General Surgery, University of the Witwatersrand, South Africa \\ ${ }^{2}$ Department of Paediatric Surgery, University of the Witwatersrand, South Africa
}

Corresponding author, email: jjacob91@gmail.com

\begin{abstract}
Summary
We present a case of a large splenic pseudocyst in a 16-year-old female, who presented with a left upper quadrant mass and features of gastric outlet obstruction. We discuss the difficulties in diagnosis, the management options and why the definitive treatment of distal pancreatectomy and splenectomy was necessary.

Keywords: splenic pseudocyst, spleen
\end{abstract}

\section{Case report}

A 16-year-old girl presented with a one-month history of a left upper abdominal mass. The patient described early satiety, nausea and occasional postprandial nonbilious vomiting. Though our patient was from North West Province, South Africa, an area endemic for echinococcosis, she did not provide a personal or family history of hydatid disease. There was no history of trauma, smoking, alcohol, illicit drug or traditional medicine use.

An approximately $200 \times 200 \mathrm{~mm}$ well-demarcated, firm, non-tender, immobile mass was palpable in the left hypochondrium. The mass crossed the midline, was not ballotable and was stationary on deep inspiration. Examination of the liver was unremarkable and there were no features of chronic liver disease or portal hypertension. General examination did not reveal pallor, lymphadenopathy or jaundice. Routine biochemistry demonstrated a normal full blood count, urea and electrolyte levels, and liver function tests. Hydatid serology was negative.

Contrast-enhanced computerised tomography (CECT) of the abdomen demonstrated a well-defined, non-enhancing, simple cystic structure measuring 186 × 176 × $226 \mathrm{~mm}$ arising from the left hypochondrium and extending to the left hemidiaphragm (Figure 1A). The pancreas and stomach were compressed and displaced. The differential diagnosis, based on CECT included a pancreatic pseudocyst, hydatid cyst and splenic cyst. A non-neoplastic aetiology was considered most likely given the history of presentation, biochemical and radiological findings.

The patient underwent an exploratory laparotomy via a left subcostal incision. Choice of incision was based on CECT findings of an isolated left upper quadrant mass. A large cyst containing approximately 4 litres of serous fluid was noted arising from the spleen, and a splenectomy with

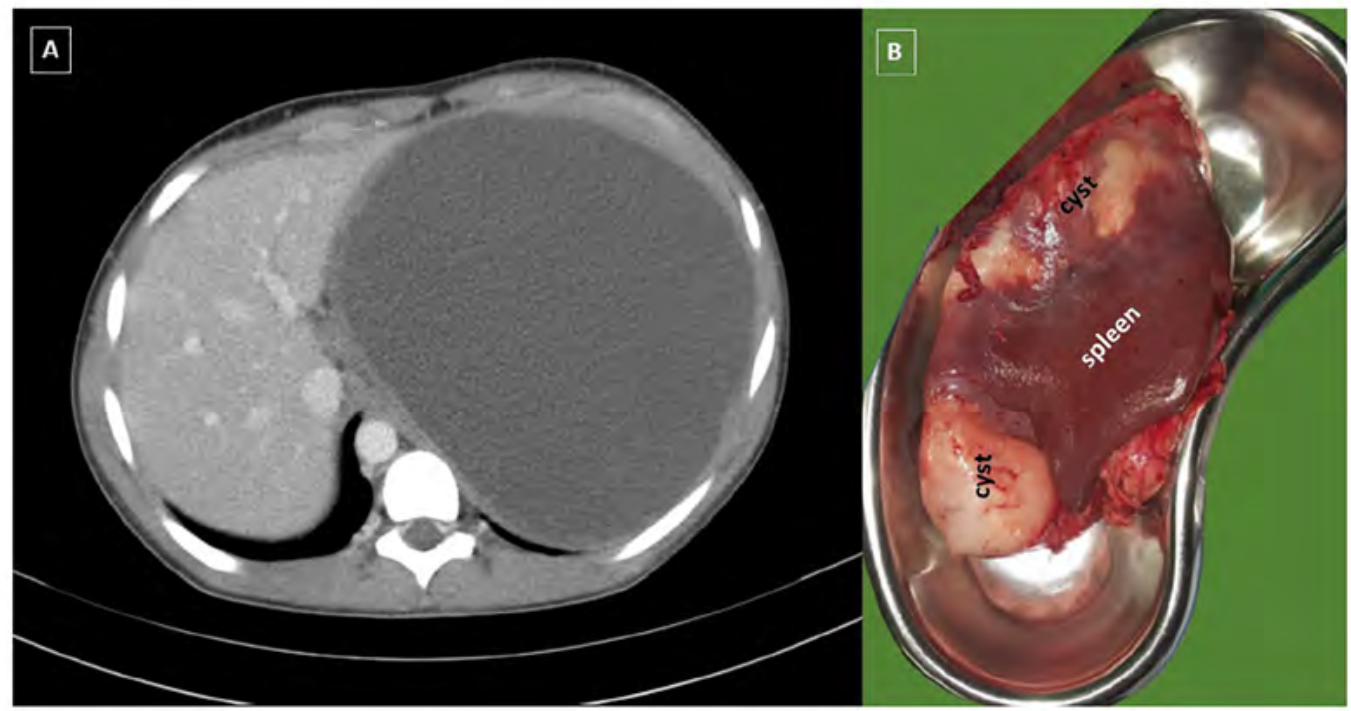

Figure 1A: Portal venous axial view of CECT demonstrating the large uniform cyst with no areas of enhancement
Figure 1B: Resected specimen demonstrating the close relationship between the cyst and the spleen 
distal pancreatectomy was performed. The surrounding viscera were well protected with dry swabs and the cyst was aspirated early in the procedure to allow better visualisation of the operative field. The aspirated fluid was not submitted for cytological analysis. Chemical and microbiological assessment of the fluid demonstrated a normal amylase level and no organisms respectively. The cyst wall was approximately $5 \mathrm{~mm}$ thick. Splenectomy and distal pancreatectomy were required to resect the cyst due to the chronically inflamed nature of the tissue surrounding the cyst and proximity of the cyst to the splenic hilum (Figure 1B). The patient had an uncomplicated postoperative recovery, receiving the pneumococcal conjugate and meningococcal vaccine prior to discharge on postoperative day 4 .

Subsequent follow-ups at 2 weeks, 6 months and 1 year post splenectomy were unremarkable, and the patient was discharged from further follow-up after reinforcing previous education on overwhelming post splenectomy infections (OPSI) and necessary follow-up care (including timing of booster vaccinations).

Histopathology revealed benign splenic parenchyma with a unilocular pseudocyst. The cyst wall compromised dense fibrous connective tissue in continuity with surrounding normal splenic parenchyma. Multinucleated giant cells, haemosiderin and haemosiderin laden macrophages were identified within the cyst. These findings are in keeping with a secondary (type II) splenic cyst.

\section{Discussion}

A large autopsy series from the University of Southern California Medical Centre revealed splenic cyst prevalence of $0.07 \% .{ }^{1}$ Martin classified splenic cysts into primary (type I) and secondary (type II), based on the presence (type I) or absence (type II) of an epithelial lining, with primary cysts further separated into parasitic and non-parasitic groups. ${ }^{2}$ Sixty per cent of all reported splenic cysts are parasitic, with the majority resulting from hydatid disease. ${ }^{3}$ Non-parasitic primary splenic cysts are usually congenital or neoplastic. Type II cysts are more commonly referred to as pseudocysts. Pseudocysts are usually due to splenic trauma, infarction or infection. ${ }^{1}$

Blunt abdominal trauma accounts for $75 \%$ of all secondary splenic cysts (pseudocysts). ${ }^{4}$ The described pathogenesis are that of an unruptured splenic haematoma which undergoes encapsulation, liquefaction, resorption and accumulation of a residue of serous fluid, macrophages, haemosiderin and cholesterol crystals. ${ }^{4,5}$ Although these findings are consistent with histopathological findings of this case, no clear history of abdominal trauma was elicited in our patient. Soria-Céspedes et al. documented a similar case of symptomatic splenic pseudocyst in the absence of clear preceding abdominal trauma in a female patient in Mexico. They proposed that degenerative changes in the epithelium of a primary cyst with atrophy and denudation may also result in the appearance of a splenic pseudocyst. ${ }^{6}$ Given the distinct absence of a history of trauma, this alternate theory may explain the pathogenesis of our patient's cystic mass.

Splenic cysts are usually asymptomatic (30-60\%) and are diagnosed incidentally. 1,7 The most common presenting symptom is vague left upper quadrant pain due to stretching of the splenic capsule. Additional symptoms manifest due to the size of the cyst and its compressive effects on the surrounding viscera. Compression of the stomach can lead to early satiety, nausea and vomiting. Constipation may be noted with compression of the transverse or descending colon. Pressure on the cardio-respiratory system may result in dyspnoea, pleuritic chest pain and persistent cough from irritation of the left hemidiaphragm. ${ }^{7}$ Very rarely, splenic cysts may present with peritonitis from rupture, haemorrhage, or infection.

The differential diagnosis of a left upper quadrant cystic mass is wide and includes benign and malignant primary retroperitoneal cysts (ranging from lymphatic malformations to cystic teratomas), omental, mesenteric, renal, hepatic, pancreatic and splenic lesions. ${ }^{8}$ Given the wide differential diagnosis, careful history taking, thorough biochemical workup and quality imaging are essential components in the formulation of a plausible differential diagnosis and appropriate management plan.

The work up of a splenic mass begins with thorough enquiry of history of trauma, travel to areas endemic for hydatid disease, and personal or family history of haematological disorders. Biochemical investigation should include a full blood count with peripheral smear and echinococcal serology. Imaging should include plain abdominal x-ray, abdominal ultrasound and CECT. Abdominal ultrasound is useful to determine if the mass is solid or cystic, the nature of flow within the splenic vein, presence of significant intra-abdominal lymphadenopathy and the relationship of the mass to surrounding structures. CECT of the abdomen is considered the current gold standard in imaging of splenic masses and is critical to medical management and surgical planning.

The management of benign splenic cysts varies from watchful waiting (cysts $<4 \mathrm{~cm}$ ), percutaneous aspiration with or without injection of a sclerosant, and a variety of surgical interventions. Percutaneous aspiration with or without sclerosants are associated with a high rate of recurrence, but may provide brief symptomatic relief. ${ }^{9}$ Given that cysts $>5 \mathrm{~cm}$ are at a high risk of rupture and lifethreatening haemorrhage, open or laparoscopic intervention is recommended in these cases. ${ }^{1,6,10}$ Negative hydatid serology does not exclude echinococcosis and every effort should be made to prevent peritoneal spillage during intervention.

Overwhelming post splenectomy infection (OPSI) is a rare entity, affecting approximately $3 \%$ of patients who undergo splenectomy. ${ }^{1}$ Mortality rates for OPSI are extremely high, reaching $50 \%$ in some studies. ${ }^{11}$ Given the potentially catastrophic effects of splenectomy and high rates of recurrence associated with cyst aspiration, various surgical techniques including partial splenectomy (preserving $>25 \%$ of spleen), total cystectomy, marsupialisation of splenic cysts and laparoscopic cyst fenestration have been described as attempts at spleen preservation. ${ }^{1,4}$ Recurrence rates from spleen preserving techniques are wide, ranging from $20 \%$ for cystectomies to $100 \%$ for cyst aspiration. ${ }^{4}$ Large splenic cysts, and more especially those involving the splenic hilum are not suitable for spleen preserving surgery due to the risk of haemorrhage. ${ }^{7}$ In such cases splenectomy remains the standard of surgical management. ${ }^{4}$

Given the size of the cyst, the patient's presenting symptoms, and radiological findings, the cyst was not initially thought to originate from the spleen and preoperative vaccinations were not administered. As such, vaccinations were administered postoperatively. In 
retrospect, any possibility of splenectomy should warrant timely administration of preoperative vaccinations. In the absence of high-risk features such as age $<5$ years, immunocompromising conditions, haematological malignancy, and previous history of sepsis, routine long-term post splenectomy antibiotic prophylaxis was not administered. ${ }^{8,10}$ Recommendations for the use of prophylactic antibiotics in post splenectomy patients vary by region. As such, implementation of locally accepted guidelines is recommended. Our patient received extensive education regarding her increased susceptibility to certain infections. Education also included the recommendations for annual influenza vaccination, regular meningococcal (every 3-5 years) and pneumococcal (every 6 years) vaccination, early health care presentation when experiencing febrile illness and education on malaria risk mitigation.

\section{Conclusion}

Left upper quadrant cystic masses are a diagnostic challenge. Primary and secondary splenic cysts should be considered as part of the differential diagnosis of such masses. The majority of primary splenic cysts are due to hydatid disease. Three-quarters of secondary splenic cysts are associated with blunt abdominal trauma. These cysts are largely asymptomatic and most often incidentally diagnosed on imaging such as abdominal ultrasonography or CECT. Asymptomatic secondary splenic cysts can be managed conservatively, with clinical follow-up and serial imaging to assess changes in size. Although various spleen-preserving options have been described for the surgical management of larger cysts in children and young adults, the possibility of splenectomy must always be contemplated. Given the possibility of splenectomy and attendant risk of OPSI, preoperative vaccination and postoperative antibiotic prophylaxis must always be considered and administered as per regional guidelines.

\section{Acknowledgements}

The authors thank Dr R Gonzalez, specialist paediatric surgeon, and Dr Andrew Nel, specialist radiologist, Klerksdorp/Tshepong Hospital Complex.

\section{Conflict of interest}

The authors declare no conflict of interest.

\section{Funding source}

None.

\section{Ethical approval}

The patient's identity has been anonymised. Confidentiality was maintained in this case report. Unconditional ethics clearance was granted by the Wits Human Research Committee (Medical) for compilation of this case report and submission. The ethics application study number is: M2005101.

\section{ORCID}

J Jacob (iD https://orcid.org/0000-0003-4903-8248

N Patel (iD https://orcid.org/ 0000-0002-8494-0360

\section{REFERENCES}

1. Ingle SB, Hinge CR, Patrike S. Epithelial cysts of the spleen: a minireview. World J Gastroenterol WJG. 2014;20(38):13899903. Available from: https://www.ncbi.nlm.nih.gov/pmc/ articles/PMC4194571/.

2. Martin JW. Congenital splenic cysts. Am J Surg. 1958;96(2): 302-8. Available from: https://www.americanjournalofsurgery. com/article/0002-9610(58)90916-4/abstract.

3. Kala PS, Azad S, Sharma T, Acharya S. Primary epithelial splenic cyst: a rare encounter. Indian J Pathol Microbiol. 2019;62(4):605. Available from: http://www.ijpmonline.org/ article. asp?issn $=0377-4929$; year $=2019$; volume $=62 ;$ issue $=4 ; \mathrm{s}$ page $=605$; epage $=607$; aulast $=$ Kala; type $=0$.

4. Geraghty M, Khan IZ, Conlon KC. Large primary splenic cyst: a laparoscopic technique. J Minimal Access Surg. 2009;5(1):14-6. Available from: https://www.ncbi.nlm.nih. gov/pmc/articles/PMC2699073/.

5. Simmons TC. Traumatic pseudocyst of the spleen. J Natl Med Assoc. 1990;82(10):727-9. Available from: https://www.ncbi. nlm.nih.gov/pmc/articles/PMC2571554/.

6. Soria-Céspedes D, Leuchter-Ibarra J, Ventura-Molina V. Nontraumatic splenic pseudocyst. Case report. Cir Cir. 2011;79(6):553-6.

7. Galyfos G, Touloumis Z, Palogos K, et al. Oversized pseudocysts of the spleen: report of two cases. Int J Surg Case Rep. 2014;5(2):104-7. Available from: https://linkinghub. elsevier.com/retrieve/pii/S2210261213003337.

8. Rubin LG, Schaffner W. Care of the asplenic patient. N Engl J Med. 2014;371(4):349-56. https://doi.org/10.1056/ NEJMcp1314291.

9. Verma A, Yadav A, Sharma S, et al. A rare splenic pseudocyst. J Surg Case Rep. 2013;2013(9). Available from: https://www. ncbi.nlm.nih.gov/pmc/articles/PMC3813775/.

10. Buzelé R, Barbier L, Sauvanet A, Fantin B. Medical complications following splenectomy. J Visc Surg. 2016;153(4):277-86. Available from: http://www.science direct.com/science/article/pii/S1878788616300297.

11. Patel N, Nicola A, Bennet P, et al. Paediatric splenectomy: the Johannesburg experience. South Afr J Child Health. 2018;12(1):21-3. Available from: http://www.scielo.org.za/ scielo.php?script $=$ sci_abstract\&pid $=$ S1999-767120180 00100005\&lng $=$ en\&nrm $=$ iso\&tlng $=$ en. 